\title{
Perturbation Approach to Reconstructing Deformations in a Coaxial Cylindrical Waveguide
}

\author{
M. Dalarsson, S. M. H. Emadi, and M. Norgren \\ Department of Electromagnetic Engineering, School of Electrical Engineering, KTH Royal Institute of Technology, \\ 10044 Stockholm, Sweden \\ Correspondence should be addressed to M. Dalarsson; mardal@kth.se
}

Received 20 January 2015; Accepted 4 June 2015

Academic Editor: Franklin A. Mendivil

Copyright (C) 2015 M. Dalarsson et al. This is an open access article distributed under the Creative Commons Attribution License, which permits unrestricted use, distribution, and reproduction in any medium, provided the original work is properly cited.

We study a detection method for continuous mechanical deformations of coaxial cylindrical waveguide boundaries, using perturbation theory. The inner boundary of the waveguide is described as a continuous PEC structure with deformations modeled by suitable continuous functions. In the present approach, the computation complexity is significantly reduced compared to discrete conductor models studied in our previous work. If the mechanically deformed metallic structure is irradiated by the microwave fields of appropriate frequencies, then, by means of measurements of the scattered fields at both ends, we can reconstruct the continuous deformation function. We apply the first-order perturbation method to the inverse problem of reconstruction of boundary deformations, using the dominant TEM-mode of the microwave radiation. Different orders of Tikhonov regularization, using the L-curve criterion, are investigated. Using reflection data, we obtain reconstruction results that indicate an agreement between the reconstructed and true continuous deformations of waveguide boundaries.

\section{Introduction}

Power transformers are fundamental components of an electric power grid that require careful monitoring and fault assessment. Mechanical deformations of power transformer windings, mainly due to the heavy mechanical forces from short-circuit currents, increase the risks of serious electrical power outages in the grid. In order to reduce the risks, it is of interest to investigate suitable online early detection methods for local mechanical winding deformations. One available method to diagnose various degradation phenomena in power transformers is the frequency response analysis (FRA) method. It is, however, only applicable when a transformer is disconnected from the power grid. FRA has been proposed for detection of winding deformations [1], but in order to reduce the risks of power outages, it is desirable to develop online methods that are applicable when the transformer is connected to the power grid. The present authors studied an online method, using microwave antennas inside the transformer, to reconstruct the radial positions of individual winding segments in $[2,3]$. In these papers, the reconstruction of the locations of the individual conductors from synthetic measurement data was performed by means of an optimization method, using only up to ten winding segments or turns, in order to reduce the computational complexity. In [4], elliptic, and more importantly, waveshaped mechanical deformations were studied. It was found that the wave-shaped deformations (to the first order of approximation) can be reduced to radial displacements such as those described in $[2,3]$, showing that essentially the same mathematical tools can be used to cover a broad range of deformations of individual conductors. It should however be noted that, unlike the approach in the present paper, the analysis in [2-4] uses a discrete conductor model with a number of individual conductors being treated as obstacles in the waveguide and where the mode matching technique is used to handle a limited number of such discrete obstacles. A more realistic number of winding segments, however, make a full-scale numerical model in the optimization procedure prohibitively complex. As the primary interest of our study is the detection of small winding deformations, it is of interest to study whether inversion methods, based on weak scattering, can be used for reconstruction of transformer winding deformations. A step towards such a method was taken in [5], 
where the transformer winding was not considered in detail but instead modeled as equivalent outer boundary surfaces, whose shape was to be reconstructed. In [5] a parallelplate waveguide model was assumed, with a piecewise linear shape of the continuous winding deformation. This study was extended in [6], which is similar to the approach pursued here. In the present paper we use the same basic mathematical formalism as in [6], but unlike the treatment in [6] where we used the approximate parallel-plate waveguide model, here we employ the more realistic coaxial waveguide model together with improved numerical techniques, both of which contribute to more accurate reconstruction results in the present paper. Although the present paper can be seen as an extended and improved version of the approximate treatment employed in [6], the abovementioned model change and numerical improvements provide an essentially novel approach to the problem of reconstruction of deformations in the lower coaxial waveguide boundary. Thus we pursue the investigations reported in $[5,6]$ further by considering a continuous axially symmetric deformation in a coaxial waveguide model of the power transformer winding structure. The inverse problem of reconstructing deformations in the lower coaxial waveguide boundary is solved using a simple and computationally efficient firstorder perturbation method. We use synthetic measurement data from the commercial FEM program HFSS to test the model.

\section{Problem Formulation and Scattering Analysis}

We assume an axially symmetric coaxial waveguide scattering configuration, oriented along the $z$-axis, as shown in Figure 1. The inner boundary cylinder is located at $r=r_{0}$ while the outer boundary cylinder is located at $r=r_{0}+a$ giving the radial width of the unperturbed cavity equal to $a$. In the context of a power transformer, the outer boundary represents the transformer tank wall while the lower boundary represents the outermost layer of the winding structure. The winding structure is thus described as an equivalent PEC surface. Using anisotropic boundary conditions [7], it is possible to formulate a more realistic approach to model the winding. Although a realistic transformer is filled with oil and uses also paper and pressboard insulation, in the present paper we assume that the medium inside the waveguide is air (or vacuum). At the inner boundary cylinder along the section $z_{1}<z<z_{2}$ there is a local deformation described by

$$
\begin{aligned}
r & =r_{0}+a g(z) \quad \text { with } \max |g(z)| \ll 1, \\
g\left(z_{1}\right) & =g\left(z_{2}\right)=0 .
\end{aligned}
$$

The inverse problem here is to reconstruct $g(z)$ in the estimation region $z_{1}<z<z_{2}$ using scattering data obtained when the waveguide is excited from both ends. In order to focus the present study on the primary scattering mechanism, due to the local deformation of the lower boundary, we assume that there are no reflections from the ends of the waveguide.

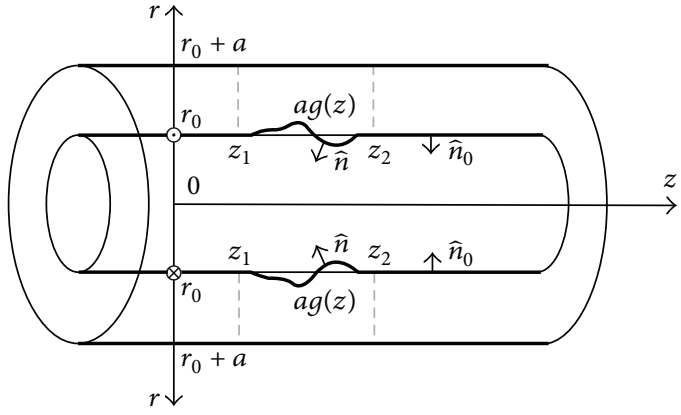

FIgURE 1: Geometry of the coaxial waveguide model.

2.1. The Direct Scattering Problem. We restrict our analysis to the TM-modes only and to mechanical deformations that possess cylindrical symmetry (i.e., are independent on the azimuthal angle $\varphi$ ). For the sake of convenience, we consider only the $\mathrm{TM}_{m}$-modes and in particular the lowest TEMmode with $m=0$. It is also possible to study the $\mathrm{TE}_{m}$-modes in the coaxial structure in a fully analogous way, which will be the subject of our continued efforts. In this case, we can use the analytic solutions for the dominant zeroth-order TEMmode $(m=0)$ in the coaxial waveguide, which propagates at all frequencies,

$$
\begin{aligned}
\mathbf{E}_{0} & =E_{0 r}\left(r_{0}\right) \frac{r_{0}}{r} \widehat{\mathbf{r}} \propto \psi_{0}(r), \\
\mathbf{H}_{0} & =H_{0 \varphi}\left(r_{0}\right) \frac{r_{0}}{r} \widehat{\boldsymbol{\varphi}} \propto \psi_{0}(r),
\end{aligned}
$$

as well as the Bessel function solutions [8] for higher-order TM-modes $(m \geq 1)$,

$$
\begin{aligned}
\mathbf{E}_{m} & =E_{m r} \widehat{\mathbf{r}} \propto \psi_{m}(r), \\
\mathbf{H}_{m} & =H_{m \varphi} \widehat{\boldsymbol{\varphi}} \propto \psi_{m}(r), \\
\psi_{m}(r) & =A_{m}\left[J_{1}\left(k_{T m} r\right)-\frac{J_{0}\left(k_{T m} a\right)}{N_{0}\left(k_{T m} a\right)} N_{1}\left(k_{T m} r\right)\right],
\end{aligned}
$$

with asymptotic expansion formula for the $m$ th wave number $k_{T m}$ given by [8]

$$
\begin{aligned}
& k_{T m} a=\frac{m \pi}{\alpha-1}-\frac{1}{8 \alpha} \cdot \frac{\alpha-1}{m \pi}+\left[\frac{25\left(\alpha^{3}-1\right)}{6(4 \alpha)^{3}(\alpha-1)}-\frac{1}{(8 \alpha)}\right] \\
& \cdot \frac{(\alpha-1)^{3}}{m^{3} \pi^{3}} \\
& -\left[\frac{1073\left(\alpha^{5}-1\right)}{5(4 \alpha)^{5}(\alpha-1)}-\frac{25\left(\alpha^{3}-1\right)}{12 \alpha(4 \alpha)^{3}(\alpha-1)}+\frac{2}{(8 \alpha)^{3}}\right] \\
& . \frac{(\alpha-1)^{5}}{m^{5} \pi^{5}}+\cdots, \quad m=1,2,3, \ldots,
\end{aligned}
$$

where $\alpha=1+a / r_{0}>1$. Here, we note that the longitudinal component of the electric field $E_{z}$ is nonzero only for $m \geq 1$. 
2.1.1. Perturbation Method. The inversion scheme (Section 2.2) is based on the solution of the direct scattering problem using a boundary perturbation method for waveguides, similar to the one used in [9]. We first rewrite (1) as follows:

$$
r-r_{0}-a \delta g(z)=0
$$

Here, $\delta$ is just a book-keeping parameter to be set equal to unity $(\delta=1)$ after the perturbation analysis. The outwardly directed surface unit normal then becomes

$$
\widehat{\mathbf{n}}=-\frac{\nabla[r-a \delta g(z)]}{|\nabla[r-a \delta g(z)]|}=\frac{-\widehat{\mathbf{r}}+a \delta(d g / d z) \widehat{\mathbf{z}}}{\sqrt{1+a^{2} \delta^{2}(d g / d z)^{2}}} .
$$

The boundary condition at the perturbed metallic boundary is

$$
\widehat{\mathbf{n}} \times \mathbf{E}=\frac{1}{j \omega \epsilon} \widehat{\mathbf{n}} \times(\nabla \times \mathbf{H})=0 .
$$

Substituting (6) in (7) and assuming TM-fields $(\mathbf{H}=H(r$, $z) \widehat{\boldsymbol{\varphi}}=H \widehat{\varphi})$, we obtain

$$
\frac{\partial H}{\partial r}-a \delta \frac{d g}{d z} \frac{d H}{d z}=0 .
$$

If we now Taylor expand $H(r, z)$ in the $r$-variable about $r=$ $r_{0}$, we obtain

$$
H(r, z)=\sum_{p=0}^{\infty} \frac{\partial^{p} H}{\partial r^{p}}\left(r_{0}, z\right) \frac{\left(r-r_{0}\right)^{p}}{p !} .
$$

Substituting (9) into (8) and using (5), we obtain up to the first order in $\delta$ as follows:

$$
\begin{aligned}
& \frac{\partial H}{\partial r}\left(r_{0}, z\right)+a \delta\left[g(z) \frac{\partial^{2} H}{\partial r^{2}}\left(r_{0}, z\right)-\frac{d g}{d z} \frac{\partial H}{\partial z}\left(r_{0}, z\right)\right] \\
& \quad=0 .
\end{aligned}
$$

Next, we develop $H(r, z)$ into a perturbation series as follows:

$$
H(r, z)=\sum_{m=0}^{\infty} H_{m}(r, z) \delta^{m} .
$$

Substituting (11) into (10) and neglecting terms of order $\delta^{2}$ and higher, we obtain

$$
\begin{aligned}
& \sum_{m=0}^{\infty} \delta^{m}\left\{\frac{\partial H_{m}}{\partial r}\left(r_{0}, z\right)\right. \\
& \left.+a \delta\left[g(z) \frac{\partial^{2} H_{m}}{\partial r^{2}}\left(r_{0}, z\right)-\frac{d g}{d z} \frac{\partial H_{m}}{\partial z}\left(r_{0}, z\right)\right]\right\} \\
& \quad=\frac{\partial H_{0}}{\partial r}\left(r_{0}, z\right)+\delta\left\{\frac{\partial H_{1}}{\partial r}\left(r_{0}, z\right)\right. \\
& \left.+a\left[g(z) \frac{\partial^{2} H_{0}}{\partial r^{2}}\left(r_{0}, z\right)-\frac{d g}{d z} \frac{\partial H_{0}}{\partial z}\left(r_{0}, z\right)\right]\right\} .
\end{aligned}
$$

Here, the terms of each order in $\delta$ must be equal to zero, which gives

$$
\begin{aligned}
& \frac{\partial H_{0}}{\partial r}\left(r_{0}, z\right)=0, \\
& \frac{\partial H_{1}}{\partial r}\left(r_{0}, z\right) \\
& \quad+a\left[g(z) \frac{\partial^{2} H_{0}}{\partial r^{2}}\left(r_{0}, z\right)-\frac{d g}{d z} \frac{\partial H_{0}}{\partial z}\left(r_{0}, z\right)\right]=0 .
\end{aligned}
$$

As can be seen from (14), the boundary perturbation results are evaluated at the unperturbed boundary $(g(z)=0)$ with $r=r_{0}$. For the first-order perturbed fields, we can use the Maxwell equation $\nabla \times \mathbf{H}_{1}=j \omega \epsilon \mathbf{E}_{1}$ together with (14) to obtain

$$
\begin{aligned}
\widehat{\mathbf{n}}_{0} & \times \mathbf{E}_{1}\left(r_{0}, z\right) \\
& =\frac{a}{j \omega \epsilon}\left[\frac{d g}{d z} \frac{\partial H_{0}}{\partial z}\left(r_{0}, z\right)-g(z) \frac{\partial^{2} H_{0}}{\partial r^{2}}\left(r_{0}, z\right)\right] \widehat{\boldsymbol{\varphi}} .
\end{aligned}
$$

Assuming now orthonormal TM-modes of the form

$$
\begin{aligned}
\mathbf{H}_{t m}(r) & =\psi_{m}(r) \hat{\boldsymbol{\varphi}}, \\
\mathbf{E}_{t m}(r) & =\frac{k_{z m}}{\omega \epsilon} \psi_{m}(r) \widehat{\mathbf{r}},
\end{aligned}
$$

we obtain

$$
\int_{r_{0}}^{r_{0}+a}\left(\mathbf{E}_{t m} \times \mathbf{H}_{t m}\right) \cdot \widehat{\mathbf{z}} 2 \pi r d r=\frac{k_{z m}}{\omega \epsilon}=Z_{m}
$$

which is valid for all modes. For the zeroth-order field with $m=0$, we have

$$
H_{0}(r, z)= \pm c_{0}^{ \pm} \psi_{0}(r) \mathrm{e}^{\mp \mathrm{j} k z}= \pm c_{0}^{ \pm} \psi_{0}(r, z),
$$

where we note that

$$
\begin{aligned}
k_{z 0} & =k \\
\psi_{0}(r) & =\frac{1}{\sqrt{2 \pi \ln \alpha}} \cdot \frac{1}{r} .
\end{aligned}
$$

Substituting (18) into (15), we obtain

$$
\widehat{\mathbf{n}}_{0} \times \mathbf{E}_{1}\left(r_{0}, z\right)=-\frac{a}{\omega \epsilon} c^{ \pm}\left[k \frac{d g}{d z} \pm \frac{2 \mathrm{j}}{r_{0}^{2}} g\right] \psi_{0}\left(r_{0}\right) \mathrm{e}^{\mp \mathrm{j} k z} \widehat{\boldsymbol{\varphi}}
$$

The results (17) and (20) are input to the excitation theorem, described in [10]. With no free currents $(\mathbf{J}=0)$, the excitation theorem becomes as follows:

$$
d_{m}^{ \pm}=\frac{1}{2 Z_{m}} \int_{\text {aperture }}\left[\mathbf{E} \times \mathbf{H}_{m}^{\mp} \mathrm{e}^{ \pm j k_{z m} z}\right] \cdot \widehat{\mathbf{n}}_{A} d A,
$$

where $\mathbf{E}$ is the exact tangential electric field in the "aperture" at $r=r_{0}$ between $z_{1}$ and $z_{2}$ and $\widehat{\mathbf{n}}_{A}$ is the (unperturbed) outward normal of the aperture surface $\left(\widehat{\mathbf{n}}_{A}=\widehat{\mathbf{n}}_{0}\right)$. The "aperture" between $z_{1}$ and $z_{2}$ in the inner wall of the waveguide $\left(r=r_{0}\right)$ serves as a source of the perturbation 
fields. Using now $d A=2 \pi r_{0} d z, \widehat{\mathbf{n}}_{A}=-\widehat{\mathbf{r}}$, and the fact that $\mathbf{H}_{t m}^{\mp}\left(r_{0}\right)$ is not $z$-dependent, we can rewrite (21) as follows, for $m=0$ and TM-modes:

$$
\begin{aligned}
d_{0}^{ \pm}= & \frac{\omega \epsilon}{2 k_{z m}} 2 \pi r_{0}\left[\mathbf{H}_{t 0}^{\mp}\left(r_{0}\right)\right] \\
& \cdot \int_{z_{1}}^{z_{2}} \widehat{\mathbf{n}}_{0} \times \mathbf{E}_{1}\left(r_{0}, z\right) \mathrm{e}^{ \pm \mathrm{j} k z} d z .
\end{aligned}
$$

Using further

$$
\mathbf{H}_{t 0}\left(r_{0}\right)=\psi_{0}\left(r_{0}\right) \hat{\boldsymbol{\varphi}}
$$

and substituting (20) into (22), we obtain

$$
\begin{aligned}
d_{0}^{+}= & 2 \pi r_{0}\left(\frac{a}{2 k}\right) c_{0}^{ \pm}\left[\psi_{0}\left(r_{0}\right)\right]^{2} \\
& \cdot \int_{z_{1}}^{z_{2}}\left[k \frac{d g}{d z} \pm \frac{2 \mathrm{j}}{r_{0}^{2}} g\right] \mathrm{e}^{\mathrm{j} k z(1 \mp 1)} d z, \\
d_{0}^{-}= & -2 \pi r_{0}\left(\frac{a}{2 k}\right) c_{0}^{ \pm}\left[\psi_{0}\left(r_{0}\right)\right]^{2} \\
& \cdot \int_{z_{1}}^{z_{2}}\left[k \frac{d g}{d z} \pm \frac{2 \mathrm{j}}{r_{0}^{2}} g\right] \mathrm{e}^{-\mathrm{j} k z(1 \pm 1)} d z .
\end{aligned}
$$

Using here [5]

$$
\left[\begin{array}{c}
d_{0}^{+} \\
d_{0}^{-}
\end{array}\right]=\left[\begin{array}{cc}
M_{00}^{\mathrm{pp}} & M_{00}^{\mathrm{pm}} \\
M_{00}^{\mathrm{mp}} & M_{00}^{\mathrm{mm}}
\end{array}\right]\left[\begin{array}{c}
c_{0}^{+} \\
c_{0}^{-}
\end{array}\right],
$$

we obtain

$$
\begin{aligned}
& M_{00}^{\mathrm{pm}}=\frac{2 \pi a r_{0}}{2 k}\left[\psi_{0}\left(r_{0}\right)\right]^{2} \int_{z_{1}}^{z_{2}}\left[k \frac{d g}{d z}-\frac{2 \mathrm{j}}{r_{0}^{2}} g\right] \mathrm{e}^{\mathrm{j} 2 k z} d z, \\
& M_{00}^{\mathrm{mp}} \\
& \quad=-\frac{2 \pi a r_{0}}{2 k}\left[\psi_{0}\left(r_{0}\right)\right]^{2} \int_{z_{1}}^{z_{2}}\left[k \frac{d g}{d z}+\frac{2 \mathrm{j}}{r_{0}^{2}} g\right] \mathrm{e}^{-\mathrm{j} 2 k z} d z .
\end{aligned}
$$

Using here $g\left(z_{1}\right)=g\left(z_{2}\right)=0$ and integrating by parts, we further obtain

$$
\begin{aligned}
& M_{00}^{\mathrm{pm}} \\
& \quad=-\mathrm{j} a \frac{1}{r_{0}^{2} \ln \lambda}\left(k r_{0}+\frac{1}{k r_{0}}\right) \frac{1}{z_{1}-z_{2}} \int_{z_{1}}^{z_{2}} g(z) \mathrm{e}^{\mathrm{j} 2 k z} d z, \\
& M_{00}^{\mathrm{mp}} \\
& =-\mathrm{j} a \frac{1}{r_{0}^{2} \ln \lambda}\left(k r_{0}+\frac{1}{k r_{0}}\right) \frac{1}{z_{1}-z_{2}} \int_{z_{1}}^{z_{2}} g(z) \mathrm{e}^{-\mathrm{j} 2 k z} d z
\end{aligned}
$$

or finally

$$
\begin{aligned}
& S_{11}(k) \approx M_{00}^{\mathrm{mp}}=-\mathrm{j} \beta(k) \mathrm{e}^{\mathrm{j} 2 k z_{1}} G^{*}(k), \\
& S_{22}(k) \approx M_{00}^{\mathrm{pm}}=-\mathrm{j} \beta(k) \mathrm{e}^{-\mathrm{j} 2 k z_{2}} G(k)
\end{aligned}
$$

being the first-order approximation for the reflection parameters $S_{11}$ and $S_{22}$. Here $k=\omega \sqrt{\epsilon_{0} \mu_{0}}$ is the vacuum wavenumber and we introduce this notation

$$
\beta(k)=\frac{a}{r_{0}^{2} \ln \alpha}\left(k r_{0}+\frac{1}{k r_{0}}\right) \frac{1}{z_{2}-z_{1}} .
$$

In (28) we also define a " $k$-transform" $G(k)$ of $g(z)$ and its complex conjugate as follows:

$$
\begin{gathered}
G(k)=\int_{z_{1}}^{z_{2}} g(z) \mathrm{e}^{\mathrm{j} 2 k z} d z, \\
G^{*}(k)=\int_{z_{1}}^{z_{2}} g(z) \mathrm{e}^{-\mathrm{j} 2 k z} d z .
\end{gathered}
$$

Corrections to the transmission parameters, for the dominant mode, are of the second order in the perturbation $g(z)$. Consider

$$
\begin{aligned}
& S_{12}=1+\mathcal{O}\left(\max \left|g^{2}\right|\right), \\
& S_{21}=1+\mathcal{O}\left(\max \left|g^{2}\right|\right) .
\end{aligned}
$$

The transmission data are therefore assumed to be too sensitive for measurement errors and consequently will not be included in the present analysis.

2.1.2. HFSS Model. In the present study, a full-wave FEM model, implemented in the commercial program HFSS, is used to generate synthetic measurement data. For simplicity, the unperturbed waveguide cavity radial size was chosen as $a=1 \mathrm{~m}$. Furthermore, we chose a geometry with $\alpha-1=a / r_{0}=$ 1. As synthetic measurement data, we computed the complex reflection parameters in the frequency range from $20 \mathrm{MHz}$ to $150 \mathrm{MHz}$ with a step size of $5 \mathrm{MHz}$ in HFSS.

2.2. The Inverse Scattering Problem. Let us now consider the perturbation function $g(z)$ with the properties given in (1). Since we require that $g\left(z_{1}\right)=g\left(z_{2}\right)=0$, any such function can be expanded into the Fourier Sine series of the form

$$
\begin{aligned}
g(z) & =\sum_{n=1}^{\infty} p_{n} \phi_{n}(z), \\
\phi_{n}(z) & =\sin \left(n \pi \frac{z-z_{1}}{z_{2}-z_{1}}\right),
\end{aligned}
$$

where we use the infinite set of mutually orthogonal sine functions $\phi_{n}(z)$ satisfying the required conditions $\phi_{n}\left(z_{1}\right)=$ $\phi_{n}\left(z_{2}\right)=0$. The coefficients $p_{n}$ in (32) are real numbers and they are not functions of $z$. If we then apply the " $k$-transform", as defined in (30), to both sides of (32), we obtain

$$
\begin{aligned}
G(k) & =\sum_{n=1}^{\infty} p_{n} \Phi_{n}(k), \\
G^{*}(k) & =\sum_{n=1}^{\infty} p_{n} \Phi_{n}^{*}(k),
\end{aligned}
$$


where

$$
\begin{aligned}
\Phi_{n}(k) & =\int_{z_{1}}^{z_{2}} \phi_{n}(z) \mathrm{e}^{\mathrm{j} 2 k z} d z \\
& =\frac{n \pi\left(z_{2}-z_{1}\right)\left(\mathrm{e}^{\mathrm{j} 2 k z_{1}}-(-1)^{n} \mathrm{e}^{\mathrm{j} 2 k z_{2}}\right)}{n^{2} \pi^{2}-4 k^{2}\left(z_{2}-z_{1}\right)^{2}}
\end{aligned}
$$

and $G(k)$ is given by (30). In order to be able to perform the inversion numerically, we approximate the continuous inverse problem with a discrete inverse problem, where the deformation $g(z)$ is expanded into a finite set of functions, whereby we truncate the infinite series in (33) to a finite number of terms denoted by $N$ as follows:

$$
\begin{aligned}
G(k) & =\sum_{n=1}^{N} p_{n} \Phi_{n}(k), \\
G^{*}(k) & =\sum_{n=1}^{N} p_{n} \Phi_{n}^{*}(k) .
\end{aligned}
$$

Since $\left\{p_{n}\right\}_{n=1}^{N}$ are real-valued, it is convenient to treat the real and imaginary parts of (35) as separate equations

$$
\begin{aligned}
& G_{R}(k)=\sum_{n=1}^{N} p_{n} \Phi_{n R}(k), \\
& G_{I}(k)=\sum_{n=1}^{N} p_{n} \Phi_{n I}(k),
\end{aligned}
$$

where $G(k)=G_{R}(k)+\mathrm{j} G_{I}(k)$ and $\Phi_{n}(k)=\Phi_{n R}(k)+$ $\mathrm{j} \Phi_{n I}(k)$, with $G_{R}(k), G_{I}(k), \Phi_{n R}(k)$, and $\Phi_{n I}(k)$ being realvalued functions of $k$. If we substitute $G(k)$ and $G^{*}(k)$ from (28) into (35), we obtain

$$
\begin{aligned}
& \beta(k) \sum_{n=1}^{N} p_{n} \Phi_{n}^{*}(k)=\mathrm{j} S_{11}(k) \mathrm{e}^{-\mathrm{j} 2 k z_{1}}, \\
& \beta(k) \sum_{n=1}^{N} p_{n} \Phi_{n}(k)=\mathrm{j} S_{22}(k) \mathrm{e}^{\mathrm{j} 2 k z_{2}},
\end{aligned}
$$

where we see that

$$
\begin{aligned}
G^{*}(k) & =\frac{\mathrm{j} S_{11}(k)}{\beta(k)} \mathrm{e}^{-\mathrm{j} 2 k z_{1}} \Longrightarrow G(k)=\frac{-\mathrm{j} S_{11}^{*}(k)}{\beta(k)} \mathrm{e}^{\mathrm{j} 2 k z_{1}}, \\
G(k) & =\frac{\mathrm{j} S_{22}(k)}{\beta(k)} \mathrm{e}^{\mathrm{j} 2 k z_{2}} .
\end{aligned}
$$

From the two results for $G(k)$, given in (38), we see that in theory measurements of $S_{11}$ and $S_{22}$ give the same $G(k)$ and consequently the same equation (35). Thus it is in principle possible to perform the reconstruction of the expansion coefficients $\left\{p_{n}\right\}_{n=1}^{N}$, using the measurements of either $S_{11}$ or $S_{22}$ (one-sided reflection data) or both $S_{11}$ and $S_{22}$ (two-sided reflection data).

In practice however, the measurements of $S_{11}$ and $S_{22}$ may provide different values of $G(k)$ due to the contributions of higher-order modes, potential measurement errors, the truncation of the infinite series, and other approximations used in the present model. Thus if we use the measured $S_{11}$ and $S_{22}$, by means of (38), we obtain the "measured" functions $G_{1 M}(k)$ and $G_{2 M}(k)$ as follows:

$$
\begin{aligned}
& G_{1 M}(k)=\frac{-\mathrm{j} S_{11}^{*}(k)}{\beta(k)} \mathrm{e}^{\mathrm{j} 2 k z_{1},}, \\
& G_{2 M}(k)=\frac{\mathrm{j} S_{22}(k)}{\beta(k)} \mathrm{e}^{\mathrm{j} 2 k z_{2}} .
\end{aligned}
$$

In the present paper we perform the reconstructions using contributions from both $S_{11}$ and $S_{22}$.

The coefficients $\left\{p_{n}\right\}_{n=1}^{N}$ are collected into the vector $\mathbf{p}$. From measurements of $G(k)$ at several values of $k$ (frequencies), the right hand side of (33) is collected into the vector $\mathbf{G}=\left[\begin{array}{ll}\mathbf{G}_{\mathbf{R}} & \mathbf{G}_{\mathbf{I}}\end{array}\right]^{\mathrm{T}}$, while the evaluations of $\Phi_{n}(k)$ are collected into the matrix with elements $\Phi_{n k}=\Phi_{n}(k)$ such that

$$
\begin{aligned}
& \boldsymbol{\Phi}=\left[\begin{array}{ll}
\boldsymbol{\Phi}_{\mathbf{R}} & \boldsymbol{\Phi}_{\mathbf{I}}
\end{array}\right]^{\mathrm{T}}, \\
& \mathbf{G}=\left[\begin{array}{ll}
\mathbf{G}_{\mathbf{R}} & \mathbf{G}_{\mathbf{I}}
\end{array}\right]^{\mathrm{T}} .
\end{aligned}
$$

The vector $\mathbf{p}$ is a solution of the least square equation $\min \|\boldsymbol{\Phi} \mathbf{p}-\mathbf{G}\|_{2}^{2}$. The inverse problem in the present paper is ill-posed, and in order to handle this illposedness, we use the Tikhonov regularization method, since it is the most commonly used method of regularization for ill-posed problems of the present type $[11,12]$. We invoke Tikhonov regularization by adding a penalty term to the above least square equation. For the $r$ th order derivative of the deformation $g(z),(33)$ implies

$$
\left\|\frac{\mathrm{d}^{r} g}{\mathrm{~d} z^{r}}\right\|_{2}^{2}=\int_{z_{1}}^{z_{2}}\left(\frac{\mathrm{d}^{r} g}{\mathrm{~d} z^{r}}\right)^{2} \mathrm{~d} z \propto \sum_{n=1}^{N} n^{2 r} p_{n}^{2}=\|\mathbf{L} \mathbf{p}\|_{2}^{2},
$$

where the matrix $\mathbf{L}=[\operatorname{diag}\{1,2, \ldots, N\}]^{r}$. We define $r$ as the order of the regularization, in the regularized problem

$$
\min \left\{\|\mathbf{\Phi} \mathbf{p}-\mathbf{G}\|_{2}^{2}+\lambda^{2}\|\mathbf{L} \mathbf{p}\|_{2}^{2}\right\}
$$

where $\lambda$ is the regularization parameter. The coefficient vector $\mathbf{p}$ that solves (41) is obtained from

$$
\left(\boldsymbol{\Phi}^{\mathrm{T}} \boldsymbol{\Phi}+\lambda^{2} \mathbf{L}^{\mathrm{T}} \mathbf{L}\right) \mathbf{p}=\boldsymbol{\Phi}^{\mathrm{T}} \mathbf{G} .
$$

To find appropriate values of the regularization parameter $\lambda$, we use the L-curve method [11], in which $\log _{10}\|\boldsymbol{\Phi} \mathbf{p}-\mathbf{G}\|_{2}$ and $\log _{10}\|\mathbf{L} \mathbf{p}\|_{2}$ are plotted against each other, when solving (43) parameterized by $\lambda$. The optimal $\lambda$ is estimated by using the MATLAB function corner [13] which finds the corner of a discrete L-curve using an adaptive pruning algorithm.

\section{Reconstruction Results}

In this section we present the reconstruction results for five different perturbation shape functions: (a) continuous 


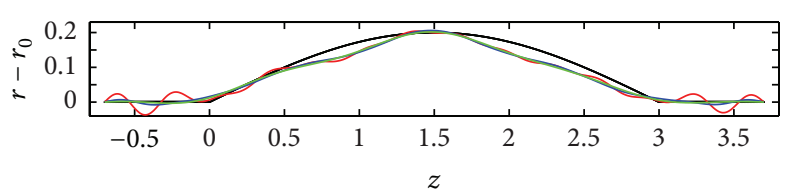

(a)

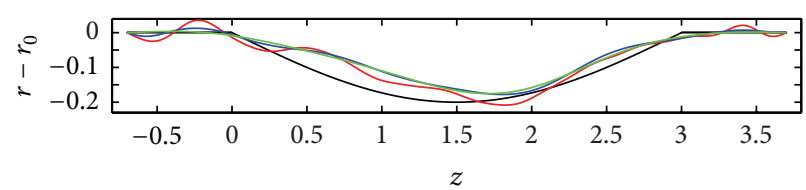

(b)

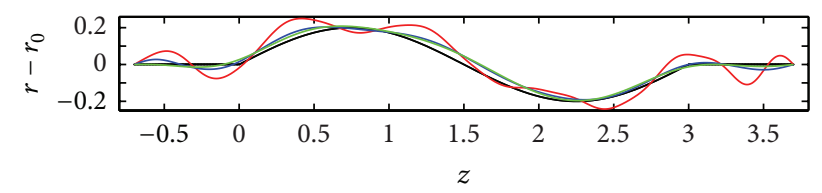

(c)

Figure 2: Reconstruction results for $r=0$ and (a) 20\% intrusion, (b) 20\% extrusion, and (c) 20\% intrusion and extrusion. In all three graphs, the true shape is represented by the black line and the reconstructed shapes are represented by the red $(r=0)$, blue $(r=1)$, and green $(r=2)$ lines.

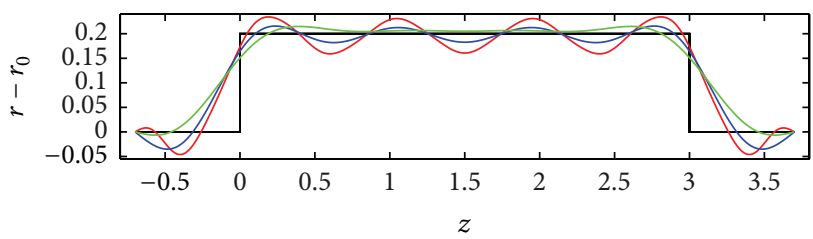

(a)

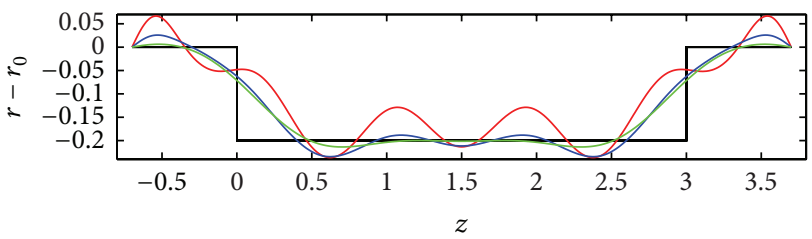

(b)

FIGURE 3: Reconstruction results for $r=0$ and (a) 20\% square intrusion and (b) 20\% square. In both graphs, the true shape is represented by the black line and the reconstructed shapes are represented by the red $(r=0)$, blue $(r=1)$, and green $(r=2)$ lines.

intrusion, (b) continuous extrusion, (c) continuous intrusion/extrusion, (d) discontinuous intrusion, and (e) discontinuous extrusion. In all the reconstruction examples, we use $N=27, r_{0}=1 \mathrm{~m}$, and $a=1 \mathrm{~m}$ for the undistorted part of the waveguide. The lengths of the extrusion/intrusions in the examples below are $3 \mathrm{~m}$ and $1.5 \mathrm{~m}$, while the estimation region is chosen larger, $4.4 \mathrm{~m}$ long in all cases. In Figure 2, we present the reconstruction results for cases (a)-(c) for the zeroth order of regularization $(r=0)$ and two higher orders of regularization $(r=1,2)$. For the continuous perturbations, we have used the function

$$
g(z)=D \sin \left(\frac{n \pi z}{b}\right)
$$

where $n=1, D=0.2$, and $b=3 \mathrm{~m}$ for the intrusion having a maximum of $20 \%$ of the undistorted waveguide width, as described in Figure 2(a), and $n=1, D=-0.2$, and $b=3 \mathrm{~m}$ for the extrusion having a maximum of $20 \%$ of the undistorted waveguide width, as described in Figure 2(b), as well as $n=$ $2, D=0.2$, and $b=3 \mathrm{~m}$ for the intrusion/extrusion, as described in Figure 2(c). In Figure 2, the true shapes are represented by black lines while the reconstructed shapes are represented by red $(r=0)$, blue $(r=1)$, and green $(r=2)$ lines.

In Figure 3, the reconstruction results for cases (d)-(e) are presented. The discontinuous perturbation is described by the usual rectangular function, with $D=0.2$ and $b=3 \mathrm{~m}$ for the intrusion in Figure 3(a) and $D=-0.2$ and $b=3 \mathrm{~m}$ for the extrusion in Figure 3(b). Also in this figure, the true shapes are represented by black lines while the reconstructed shapes are represented by red $(r=0)$, blue $(r=1)$, and green $(r=2)$ lines.

From Figures 2 and 3 we see that the results obtained using the zeroth-order regularization display relatively large deviations between the reconstructed and true shapes. The corresponding reconstruction results, obtained using the first and especially second order of regularization, are better and can be considered accurate enough for potential diagnostic purposes. The present investigation indicates that higher orders of regularization $r>2$ do not considerably improve the accuracy, which indicates that the optimal regularization order is likely to be of the order $r=\{1,2\}$. The reconstruction method works robustly for different heights of the perturbation. Figure 4 shows the reconstruction results for three different heights of continuous intrusions (30\%, 40\%, and $50 \%)$. The true shapes are represented by the black lines and the reconstructed shapes by the blue $(r=1)$ lines. It turns out that the reconstruction results using $r=1$ regularization accurately reflect the different magnitudes of the the deformation function. It is also interesting to point out here that the perturbation method works well even for deformations that extend to half of the waveguide width. Figure 5 illustrates the same principle, but this time for three different heights of discontinuous extrusions (10\%, 20\%, and $30 \%)$.

Finally, in Figures 6 and 7 the method is illustrated for $20 \%$ intrusions and extrusions but with shorter deformation lengths than in the previous examples. In Figures 6 and 7 


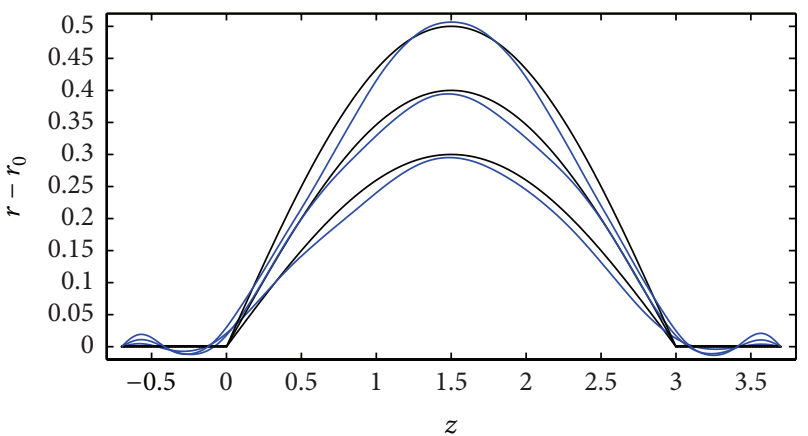

FIGURE 4: Reconstruction results for different heights of sinusoidal intrusions (30\%, 40\%, and 50\%). The true shapes are represented by the black lines and the reconstructed shapes by the blue $(r=1)$ lines.

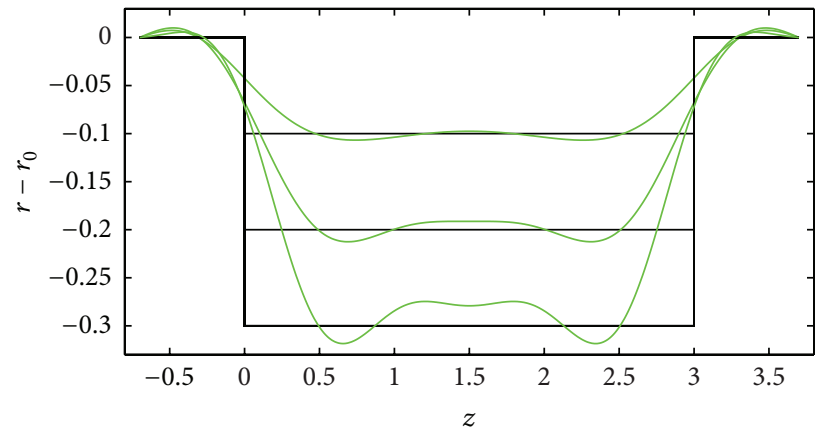

FIGURE 5: Reconstruction results for different heights of square extrusions (10\%, 20\%, and 30\%). The true shapes are represented by the black lines and the reconstructed shapes by the green $(r=2)$ lines.

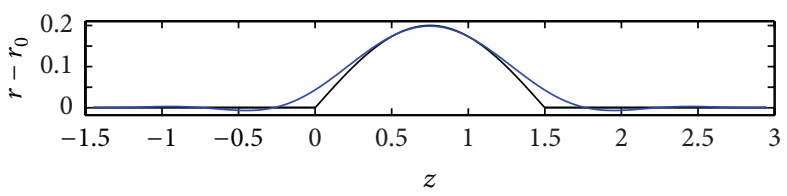

(a)

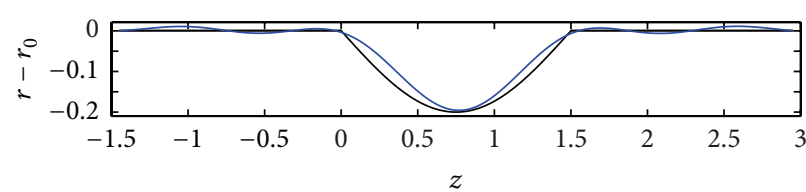

(b)

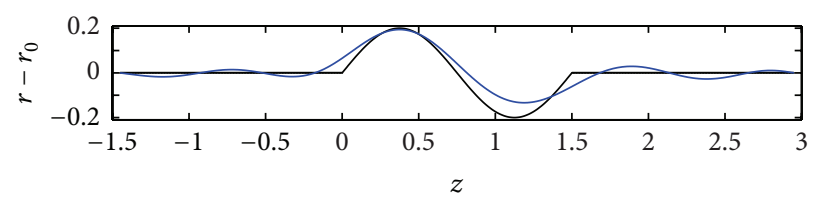

(c)

FIGURE 6: Reconstruction results for deformation length $1.5 \mathrm{~m}$ and (a) 20\% intrusion, (b) 20\% extrusion, and (c) 20\% intrusion and extrusion. In all three graphs, the true shape is represented by the black line and the reconstructed shapes are represented by the blue $(r=1)$ lines.

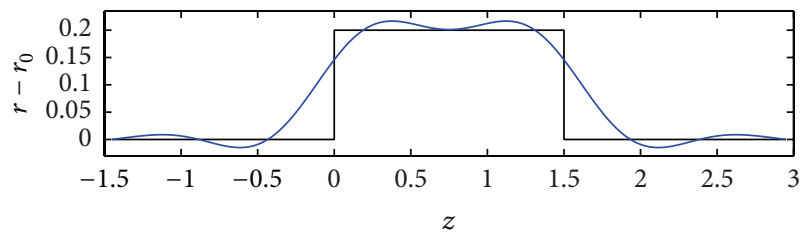

(a)

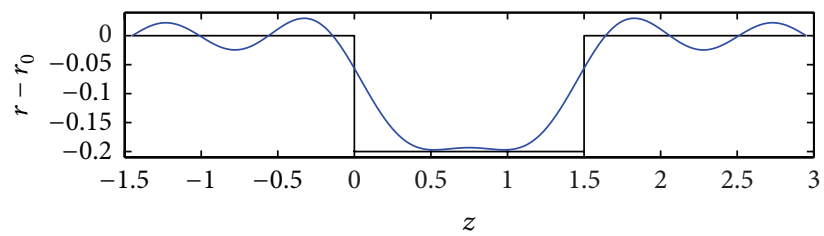

(b)

Figure 7: Reconstruction results for deformation length $1.5 \mathrm{~m}$ and (a) 20\% intrusion and (b) 20\% extrusion. In both graphs, the true shape is represented by the black line and the reconstructed shapes are represented by the blue $(r=1)$ lines. 
the deformations span a length of $1.5 \mathrm{~m}$ in the $z$-direction, instead of $3 \mathrm{~m}$ as in the previous examples. The reconstruction method performs well also for this case of shorter deformations.

It should be noted here that the high accuracy of the reconstructions in cases of continuous intrusions (see e.g., Figure 2(a)) and extrusions (Figure 2(b)) is encouraging, since, from the practical point of view, these perturbation functions are more likely to be used to model the realistic effects of heavy short-circuit forces on power transformer windings. The other studied perturbation functions are less likely to describe the realistic effects of heavy short-circuit forces, and the occurrence of such deformations would probably lead to a total failure of a power transformer.

\section{Conclusions}

A simple and computationally efficient first-order perturbation approach to the inverse problem of reconstructing deformations in a lower coaxial waveguide boundary, based on the contributions from the dominant mode only, has been investigated. Using a full-wave FEM model implemented in the commercial program HFSS, as the generator of synthetic measured reflection data, we obtained reconstruction results indicating an agreement between the reconstructed and true continuous deformations of waveguide boundaries. The cases presented in the paper, as well as other results omitted for sake of brevity, show that the method works well for continuous and discontinuous deformations and is able to distinguish between these two types of shapes. The method is also stable under different deformation heights and lengths. As a proposal for continued efforts, other regularization techniques based on Tikhonov regularization could be considered. Furthermore, improved methods to include the contributions from higher-order modes would also be of interest.

\section{Conflict of Interests}

The authors declare that there is no conflict of interests regarding the publication of this paper.

\section{Acknowledgment}

This work was funded by the Swedish Energy Agency, Project no. 34146-1.

\section{References}

[1] Y. Li, G. Liu, L. Zhang, and Z. Lin, "Transformer winding deformation diagnosis using middle band frequency response analysis," in Proceedings of the International Conference on Solid Dielectrics (ICSD '07), pp. 677-680, Winchester, UK, July 2007.

[2] M. Dalarsson, A. Motevasselian, and M. Norgren, "Online power transformer diagnostics using multiple modes of microwave radiation to reconstruct winding conductor locations," Inverse Problems in Science and Engineering, vol. 22, no. 1, pp. 84-95, 2014.
[3] M. Dalarsson, A. Motevasselian, and M. Norgren, "Using multiple modes to reconstruct conductor locations in a cylindrical model of a power transformer winding," International Journal of Applied Electromagnetics and Mechanics, vol. 41, no. 3, pp. 279291, 2013.

[4] M. Dalarsson and M. Norgren, "First-order perturbation approach to transformer winding deformations," Progress in Electromagnetics Research Letters, vol. 43, pp. 1-14, 2013.

[5] M. Norgren, M. Dalarsson, and A. Motevasselian, "Reconstruction of boundary perturbations in a waveguide," in Proceedings of the 21st International Symposium on Electromagnetic Theory (EMTS '13), pp. 934-937, Hi-roshima, Japan, May 2013.

[6] M. Dalarsson, S. M. H. Emadi, and M. Norgren, "Reconstruction of continuous deformations in a coaxial cylindrical waveguide using Tikhonov regularization," in Proceedings of the 31th URSI General Assembly and Scientific Symposium (URSI GASS '14), pp. 1-4, Beijing, China, August 2014.

[7] N. Kumar, S. K. Srivastava, and S. P. Ojha, "A theoretical analysis of the propagation characteristics of an annular circular waveguide with a helical winding as the inner cladding," Microwave and Optical Technology Letters, vol. 37, no. 1, pp. 6974, 2003.

[8] M. Abramowitz and I. A. Stegun, Handbook of Mathematical Functions, vol. 55 of Applied Mathematics Series, US Department of Commerce, National Bureau of Standards, Washington, DC, USA, 1964.

[9] H.-S. Tuan, "The radiation and reflection of surface waves at a discontinuity," IEEE Transactions of Antennas and Propagation, vol. 21, no. 3, pp. 351-356, 1973.

[10] J. D. Jackson, Classcial Electrodynamics, Wiley, New York, NY, USA, 3rd edition, 1999.

[11] P. C. Hansen and D. P. O'Leary, "The use of the L-curve in the regularization of discrete ill-posed problems," SIAM Journal on Scientific Computing, vol. 14, no. 6, pp. 1487-1503, 1993.

[12] M. Hanke, "Limitations of the $L$-curve method in ill-posed problems," BIT. Numerical Mathematics, vol. 36, no. 2, pp. 287301, 1996.

[13] P. C. Hansen, "Regularization tools version 4.0 for matlab 7.3," Numerical Algorithms, vol. 46, no. 2, pp. 189-194, 2007. 


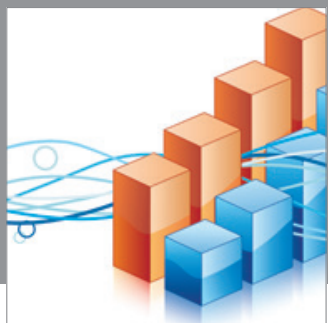

Advances in

Operations Research

mansans

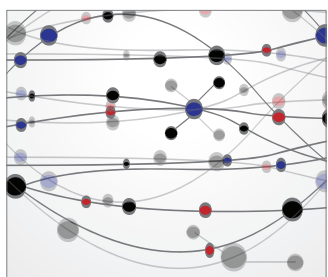

The Scientific World Journal
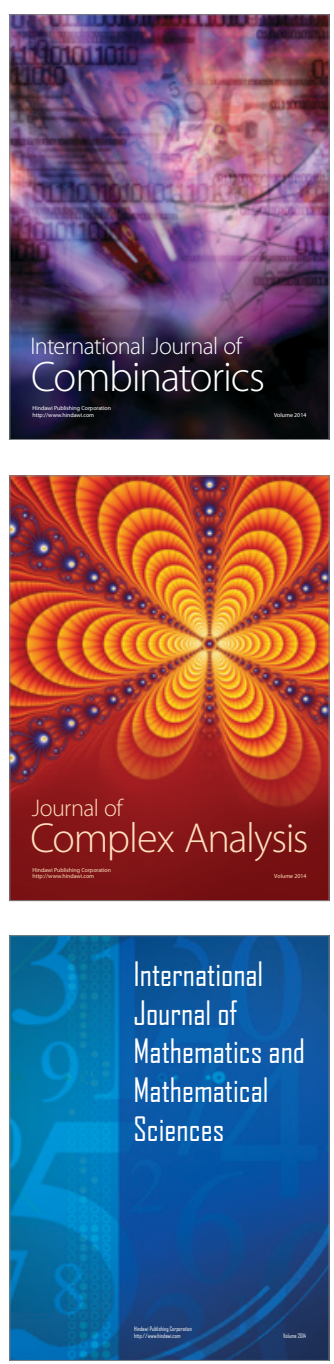
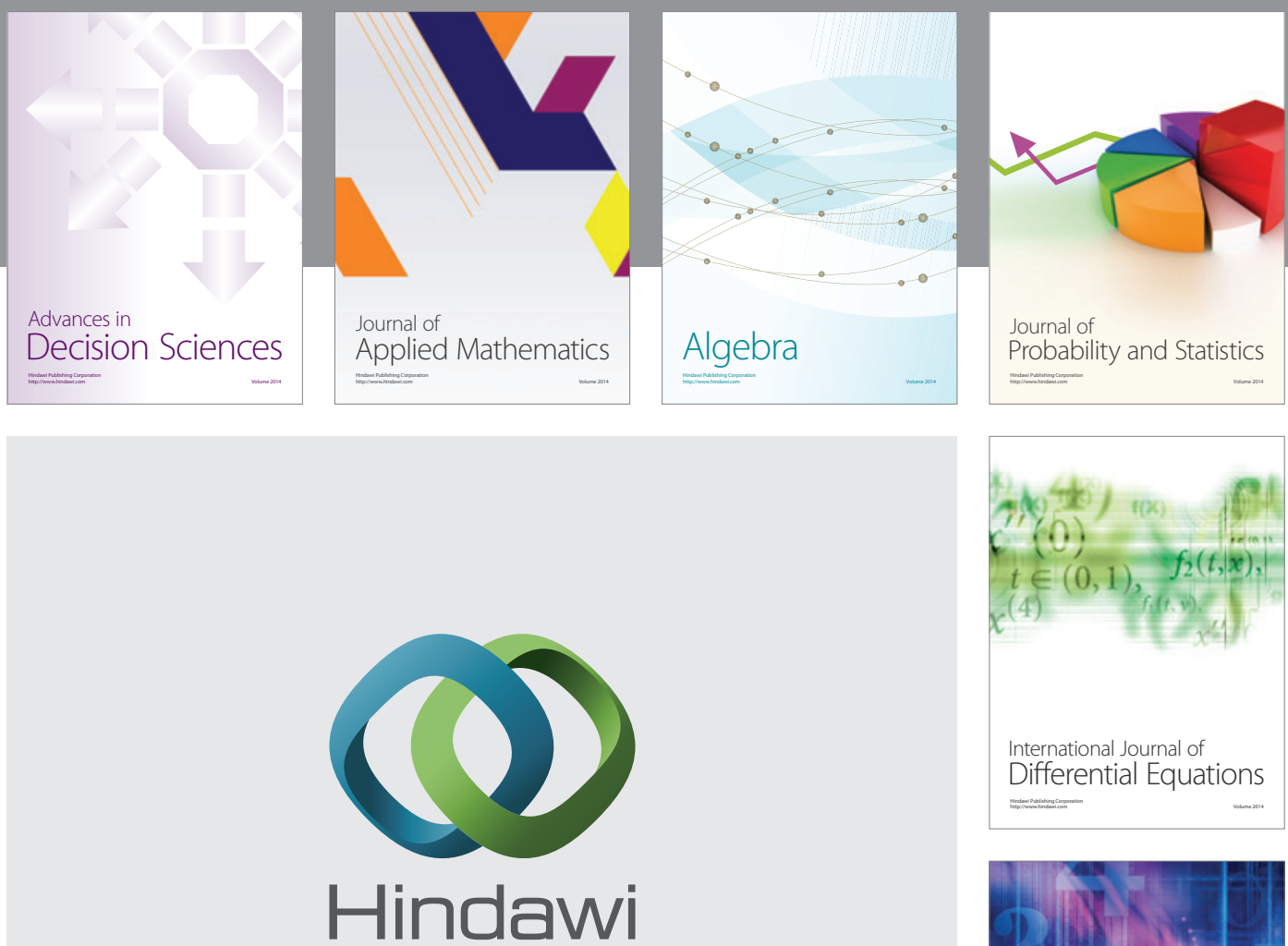

Submit your manuscripts at http://www.hindawi.com
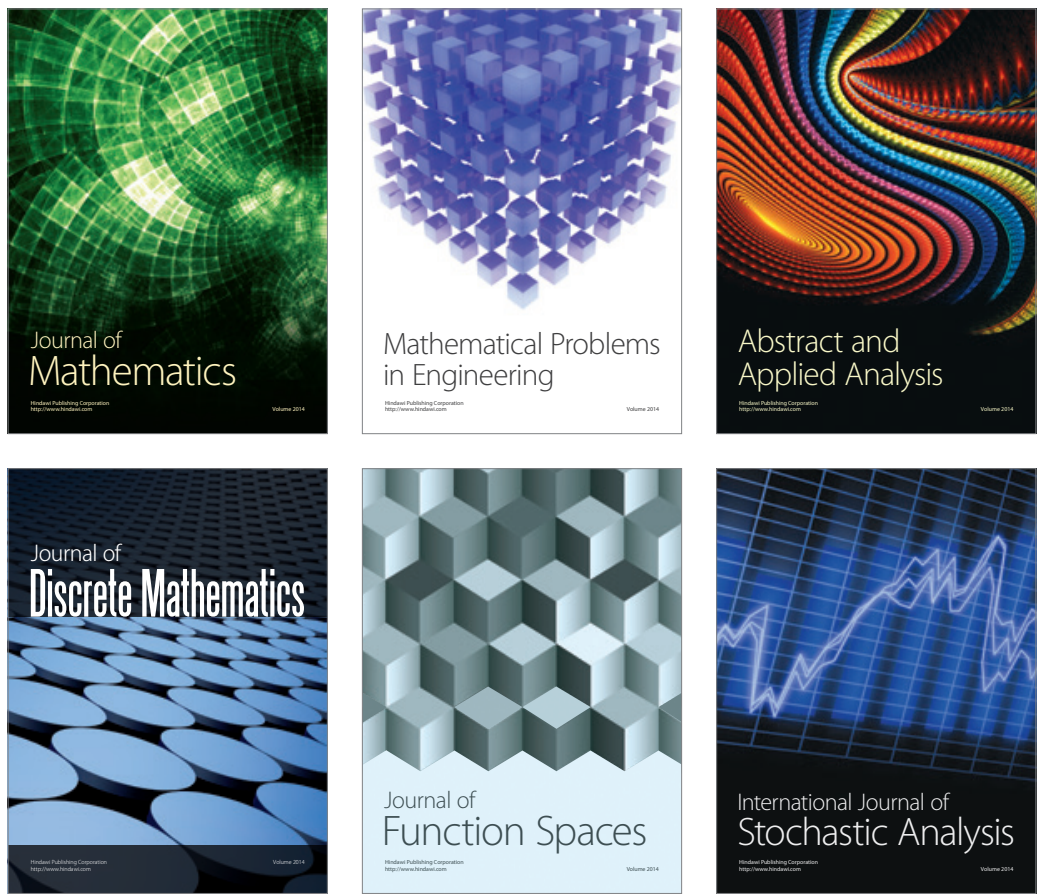

Journal of

Function Spaces

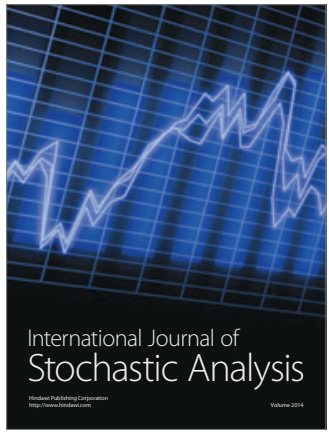

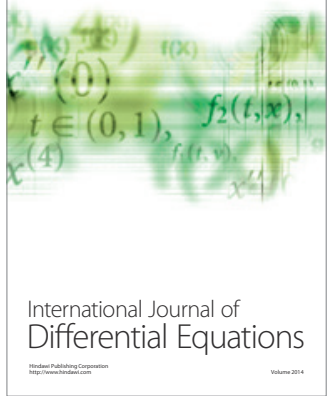
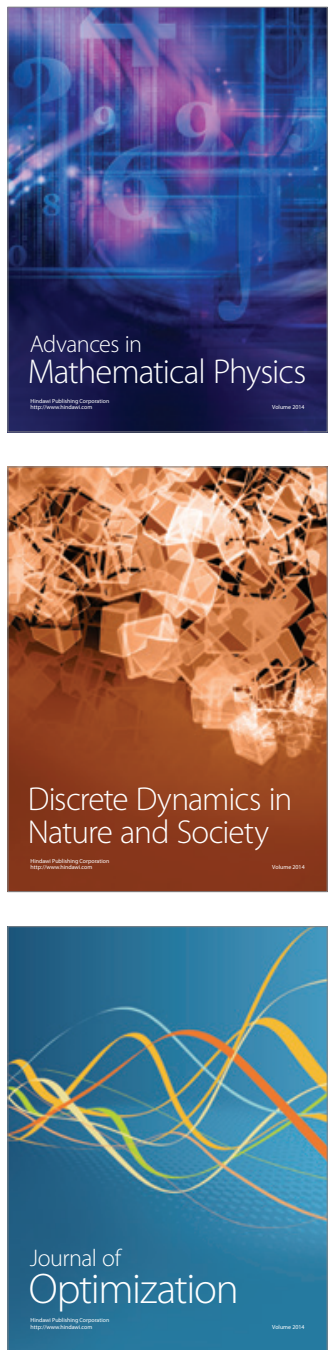